\title{
High Polygenic SNP scores do not exclude monogenic Familial Hypercholesterolaemia
}

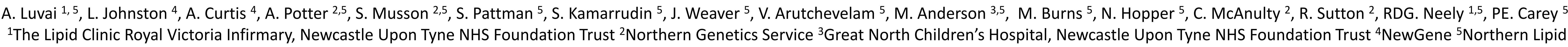
Specialists Advisory Group

\section{Introduction}

Mutations in three different genes (LDLR, APOB and PCSK9) affecting the LDL-receptor pathway are known to cause autosomal dominant Familial Hypercholesterolaemia (FH). In approximately $20 \%$ of probands with a definite clinical diagnosis of FH no mutations are detected suggesting that there may be FH causing mutations located in other genes. However it is proposed that polygenic SNP scores above the median in mutation negative patients infer a low likelihood of monogenic FH. LDL-C SNP scores in the $6^{\text {th }}-10^{\text {th }}$ decile of the distribution reported by Talmud et al ., $(2013)^{1}$ are considered to have high likelihood of polygenic aetiology.

\section{Methods}

DNA samples from probands with a Dutch Lipid Clinic Network (DLCN) score of 6 or greater were tested using a bespoke MALDI-TOF Sequenom mass spectrometry assay incorporating the low-density lipoprotein cholesterol gene score reported by Talmud et al., to distinguish patients with polygenic and monogenic hypercholesterolaemia ${ }^{1,2}$. Samples in which no $\mathrm{FH}$ causing mutations were identified underwent further analysis by Next Generation Sequencing (NGS, MiSeq/Multiplicom). Captured regions included the entire coding region and untranslated regions of LDLR, PCSK9 and APOE genes as well as a selected region of the APOB gene (Exon 26 p.3395 to p.3701). We compared the frequency of pathogenic mutations among $\mathrm{FH}$ probands in relation to deciles of polygenic SNP scores.

\section{Results}

Of the $478 \mathrm{FH}$ probands tested, $48(10.0 \%)$ had LDL-C SNP scores in the $1^{\text {st }}$ $3^{\text {rd }}$ decile indicating low likelihood of polygenic aetiology, $79(16.5 \%)$ had LDL-C SNP scores in the $4^{\text {th }} 5^{\text {th }}$ decile indicating borderline likelihood of polygenic aetiology and the vast majority 363 (73.5\%) had LDL-C SNP scores in the $6^{\text {th }}-10^{\text {th }}$ decile indicating high likelihood of polygenic aetiology (Figure 1). Of the probands with high likelihood of polygenic aetiology $68(19.2 \%)$ were mutation positive. Of the 121 mutation positive probands $68(56.2 \%)$ had a polygenic score in the $6^{\text {th }}-10^{\text {th }}$ decile suggesting high likelihood of polygenic aetiology and 49 (40.5\%) of mutation positive FH patients had polygenic SNP scores in the top 3 deciles (Figures 2/3). Four unrelated probands with the pathogenic APOE mutation (APOE c.500_502delTCC p.(Leu167del) had LDL-C SNP scores in the $6^{\text {th }}-10^{\text {th }}$ decile that would be considered compatible with polygenic hypercholesterolaemia (Table 1).

\begin{tabular}{|c|c|c|c|c|c|}
\hline Proband & APOE pathogenic variant & Gender & Age & LDL-C SNP score & LDL-C SNP decile \\
\hline NUT 157 & APOE c.500_502delTCC p.(Leu16Tdel) & $\mathrm{F}$ & 43 & 1.010 & 8th \\
\hline SUN 092 & APOE c.500_502delTCC p.(Leu16Tdel) & $\mathrm{F}$ & 45 & 1.007 & 7 th \\
\hline DUR 111 & APOE c.500_502delTCC p.(Leu16Tdel) & $\mathrm{F}$ & 32 & 1.020 & 7 th \\
\hline SUN 155 & APOE c.500_502delTCC p.(Leu16Tdel) & $\mathrm{F}$ & 51 & 1.026 & 8 th \\
\hline
\end{tabular}

Table 1: $L D L-C$ SNP scores of probands with APOE pathogenic variant

\section{Conclusion}

In our population, the probability of finding monogenic disease was two times higher among probands with LDL-C SNP scores in the $1^{\text {st }}-5^{\text {th }}$ decile compared with those in the $6^{\text {th }}-10^{\text {th }}$ decile (mutation yield $41.7 \% \mathrm{cf}$. $19.2 \%$ ). However SNP scores in the top 3 deciles are prevalent among patients with monogenic $\mathrm{FH}$ and were observed in $40.5 \%$ of this group. Mutations in the $A P O E$ gene can cause autosomal dominant Familial Hypercholesterolaemia but this gene is not routinely screened in some genetic laboratories. The four probands we identified had LDL-C SNP scores that would be considered compatible with polygenic aetiology. Genetic laboratories which do not routinely sequence the APOE gene may miss such patients and misclassify them as polygenic hypercholesterolaemia. Screening of the APOE gene is warranted in the setting of molecular diagnosis of FH along with the LDLR, APOB and PCSK9 genes. Low-density lipoprotein cholesterol gene scores do not reliably distinguish patients with monogenic familial hypercholesterolaemia from those with polygenic hypercholesterolaemia. LDL-C SNP scores should be interpreted in relation to the severity of the lipid phenotype such as age and gender specific LDL-C centiles.

Contact: Ahai.Luvai@nuth.nhs.uk

\section{References}

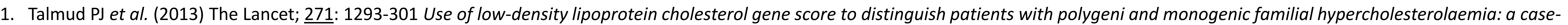
control study

2. Taylor A et al. (2010) Clin Genet 77: 572-580 Mutation detection rate and spectrum in familial hypercholesteroloaemia patients in the UK pilot cascade project 